\title{
Presentation modality and proactive interference in short-term retention using a mixed-modality distractor task*
}

\author{
RONALD H. HOPKINS $\dagger$, RICHARD E. EDWARDS, FREDERICO M. V. TAMAYO \\ MEREDITH A. HOLMAN and CHERI L. COOK \\ Washington State University, Pullman, Washington 99163
}

\begin{abstract}
Two experiments investigated the release from PI following a shift in mode of presentation of the study words. In each experiment, the interpolated task required processing of both auditory $(A)$ and visual $(V)$ information. The results were the same with mixed lists (Experiment I) and with independent groups (Experiment II): A release from PI was obtained following an $\mathrm{A}$ to $\mathrm{V}$ shift but not following a $\mathrm{V}$ to $\mathrm{A}$ shift. It was concluded that the mixed-modality filler task has functional characteristics very similar to those of an A filler and that the filler task influences the memory code for study items.
\end{abstract}

Hopkins, Edwards, and Gavelek (1971) showed that a shift in mode of presentation from visual (V) to auditory (A) produced a release from proactive interference (PI) in the paradigm developed by Wickens and his coworkers (Wickens, 1970, 1972). However, a shift in the A to V direction did not result in release from PI. These results seemed particularly important because of their potential implications for the encoding of modality information in the short-term store and because this was the only known situation in which an asymmetrical release from PI was obtained (Wickens, 1972). However, Hopkins et al used only a V filler task, and Hopkins, Edwards, and Cook (1973) subsequently showed that mode of presentation of the distractor task interacts with the presentation modality of the study items, both in determining overall retention and in producing a release from PI. In the latter study, it was found that short-term retention of $\mathrm{V}$ words is better with an $\mathrm{A}$ filler task than with an interpolated task presented in the $\mathrm{V}$ mode, but $\mathrm{A}$ words are better retained with a $\mathrm{V}$ than with an $\mathrm{A}$ filler. More importantly for present purposes, when the filler items are presented in the $\mathrm{V}$ mode, as in the earlier Hopkins et al (1971) experiments, a release from PI is obtained following a V to A shift but not following an $\mathrm{A}$ to $\mathrm{V}$ shift in the mode of presentation of the study words; with an A filler task, on the other hand, release from $\mathrm{PI}$ is obtained only for an $A$ to $\mathrm{V}$ shift in presentation modality of the study words. The experiments reported here used a mixed-mode interpolated task to explore further the role of the

*This research was supported by United States Public Health Service Grant MH 20232-01, by Basic Research Grant Award OEG-X-71-0032(508) from the United States Office of Education, and by graduate school research funds. The second author was a United States National Aeronautics and Space Administration Fellow during the conduct of this research.

$\uparrow$ Requests for reprints should be sent to Ronald H. Hopkins. Department of Psychology, Washington State Unjversity, Pullman. Washington 99163. rehearsal-prevention activity in the encoding of $\mathrm{A}$ and $\mathrm{V}$ study words.

For the filler task of the present experiments, pairs of digits were presented at a rapid rate and $S$ was to report the sum for each pair. The digits of a pair were presented simultaneously, one in the A mode and one in the $\mathrm{V}$ mode. Thus, each filler item required processing of both $A$ and $V$ informaticn. On the basis of the effects of filler mode described by Hopkins et al (1973), it seemed reasonable to assume that this type of interpolated task should interfere equally with the retention of $\mathrm{A}$ and $\mathrm{V}$ information. To the extent that a release from PI is obtained, it should be symmetrical and may be taken as evidence for differential encoding of $\mathrm{A}$ and $\mathrm{V}$ study words. If no release from PI were obtained, it could be inferred that $\mathrm{A}$ and $\mathrm{V}$ study words are similarly encoded in this situation.

\section{EXPERIMENT 1}

In addition to incorporating the mixed-mode filler task, this experiment used a procedure in which some of the study words were $A$ and some were $V$ on each trial. This mixed-list procedure has apparently never been used before with the release-from-PI paradigm. However, it has several potential advantages over the usual procedure. In addition to a reduction in the number of treatment groups required, this procedure permits the observation of certain processes in the statistically more powerful within-S design. For example, the Mode by Trials interaction, which would indicate differential rates of PI development, is difficult to detect in the usual between-S design but may be detectable in the within-S design.

\section{Method}

Each $S$ received four successive trials in a distractor-type short-term retention task. On each trial. four words wert presented for $S$ to study. followed by an interpolated task to 


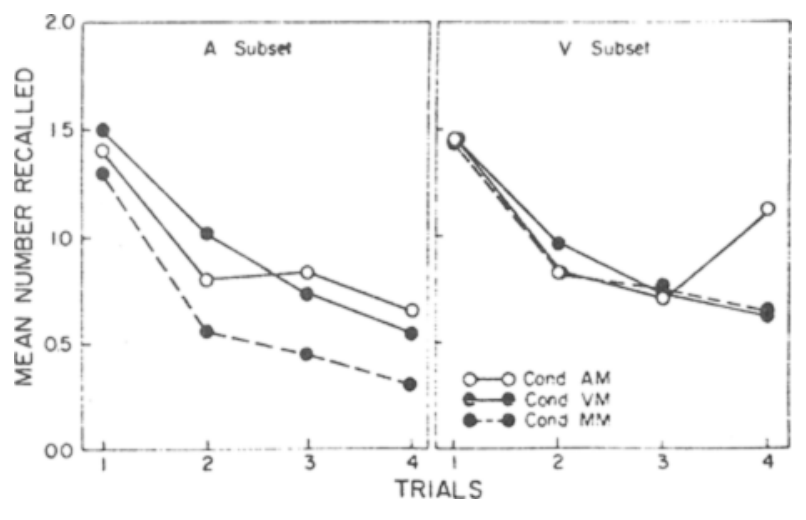

Fig. 1. Mean recall performance on Trials $1-4$ of Experiment $I$. For Trials 1.3 the $A$ and $V$ subsets were both presented in the $A$ mode in Condition AM and in the V mode in Condition VM. For all trials of Condition MM and for Trial 4 of Conditions AM and $V M$, the $A$ subset was presented in the $A$ mode and the $V$ subset in the $\mathrm{V}$ mode.

prevent rehearsal, and then recall. There were three independent groups. In Condition AM (auditory shifting to mixed presentation), all words were presented in the A mode for the first three trials; on Trial 4 two of the words were presented in the A mode and two in the V mode. Similarly, in Condition VM, all words were presented in the $V$ mode on Trials 1-3, but two were presented in the A mode and two in the $V$ mode on Trial 4. In the control condition, MM, two words were presented in the A mode and two in the $\mathrm{V}$ mode on each of the four trials.

The following describes the lists for Condition MM. The words were 16 different animal names from the Battig and Montague (1969) norms. Four different 4-tuples of these words were formed, such that the mean category frequency was approximately the same for each of the 4-tuples. Four basic lists were then prepared, with the assignment of 4-tuples to trial positions counterbalanced. Within each 4-tuple, two words were assigned to the $A$ subset and two to the $V$ subset, such that each of the following serial orders of the two subsets was used for exactly one of the 4-tuples: AVAV, VAVA, VAAV, and AVVA. Then the assignments of words to the $A$ and $V$ subsets were counterbalanced, making two forms of each basic list for a total of eight lists. Identical lists were constructed for Conditions AM and VM. The $A$ and $V$ subsets were, of course, merely a counterbalancing factor on Trials 1-3 of these latter conditions.

The interpolated task required simple addition' of two digits. One digit was presented in the $A$ mode and one in the $V$ mode, and $S$ was instructed to report the sum. The digits were selected randomly, with the restriction that none was zero. The same 32 digit pairs, 8 per trial, were used in all eight lists.

All V items were presented on a rear-projection screen by two alternating slide projectors. Auxiliary knife shutters were used to provide an exposure duration of $0.5 \mathrm{sec}$ in each case, approximately the duration of the A stimuli. The A stimuli were recorded on magnetic tape and presented via headphones. Synchronization of the $\mathrm{A}$ and $\mathrm{V}$ stimuli was provided by a digital programming device (Hopkins, 1972), using codes recorded on the unused channel of the two-channel tape deck.

The word "study" was presented at the beginning of each trial; $2 \mathrm{sec}$ later the words of a 4-tuple were presented at a rate of one word per second. The first digit pair was presented $1 \mathrm{sec}$ after the onset of the last study word; the $\mathrm{A}$ and $\mathrm{V}$ digits were presented simultaneously at a rate of one pair each $1.5 \mathrm{sec}$. The word "recall" followed the eighth digit pair by $1.5 \mathrm{sec}$, and S was allowed $3 \mathrm{sec}$ for recall. The next trial began immediately, with no intertrial interval.

Each $S$ was instructed concerning the various types of presentation and the possibility of a shift in mode of presentation and was informed that once a word had been tested it would not occur again. He was told to recall the words in the same order as they were presented and to guess if uncertain. Each $S$ was given practice on the filler items until he was completely familiar with the task; usually one trial through a list of 10 practice pairs was sufficient.

The Ss were 144 Washington State University undergraduates who participated in partial fulfillment of course requirements for introductory psychology. A block-randomized procedure was used to assign $48 \mathrm{Ss}$ to each of the three groups.

\section{Results and Discussion}

The mean numbers of words correctly recalled are presented in Fig. 1. Order of recall was ignored in scoring, and recall performance is shown separately for Subsets $\mathrm{A}$ and $\mathrm{V}$ on all trials. This method of presenting the data provides for the same maximum score of two correct in all conditions. However, it must be remembered that both subsets were presented in the $\mathbf{A}$ mode on Trials $1-3$ of Condition AM and in the V mode on Trials $1-3$ of Condition VM. Thus, there should be no difference in recall between the $A$ and $V$ subsets of these two conditions for the first three trials.

The data for the first three trials were analyzed with the item subset, condition, and trials as variables. Obviously, there was a significant decrement in recall scores across the first three trials $[F(2,282)=78.74$, $\mathrm{p}<.001$, standard error of the mean $=.108$ ], presumably due to the development of PI. The only other significant sources of variance were condition $[F(2,141)=3.24, p<.05$, standard error of the mean $=$ $.117]$, and the interaction of Item Subset by Condition $[F(2,141)=6.16, p<.005$, standard error of the mean $=.081]$. These latter two effects are apparently both due to depressed performance on $A$ items of Condition MM. Follow-up tests confirmed this, showing that recall of $\mathrm{A}$ items was lower than that of $\mathrm{V}$ items in Condition MM $[F(2,141)=13.49, p<.001$, standard error of the mean $=.081]$; however, as expected, the two subsets were not significantly different in either Condition AM or VM (both Fs $<1$ ). Although the mean recall score (pooled over trials and subsets) was slightly higher for Condition VM than for Condition AM $(X=1.05$ vs $X=1.01)$, this difference was clearly not reliable $(F<1)$. Thus, under these conditions, short-term retention is better for items presented in the $\mathrm{V}$ mode than for items presented in the A mode, but the difference is significant only in the mixed-list comparison of Condition MM. Since none of the interactions with trials was reliable, the effect of mode of presentation on the rate of PI development was not significant for either the mixed-list or homogeneous-list comparisons.

Turning to the shift data from Trial 4, a release from PI following a $V$ to $A$ shift would be indicated if the $A$ subset of Condition VM were better recalled than the A subset of Condition AM and the control condition, MM. 
It is apparent in the left panel of Fig. 1 that Trial 4 performance was not better for Condition VM than for Condition AM, though observed performance for Condition MM was somewhat lower. In fact, the effect of condition on Trial 4 recall of items from the A subset did not reach normally accepted standards of reliability $[F(2,141)=3.44, p \cong .07$, standard error of the mean $=$ $.092]$. On the other hand, a release from PI following an A to $\mathrm{V}$ shift is indicated if the $\mathrm{V}$ subset of Condition AM is better recalled than the $V$ subset of the other two conditions, and it may be seen in Fig. 1 that this was the obtained result $[F(2,141)=8.02, p<.01$, standard error of the mean $=.100$ ].

The numbers of correct responses to the filler task were also analyzed with group and trials as variables. Neither main effect nor the interaction was reliable. Overall, $89 \%$ of the filler responses were correct.

It is apparent that, under the present conditions, the release from PI following a shift in mode of presentation is asymmetrical. The release occurs for an $\mathrm{A}$ to $\mathrm{V}$ shift but not for a $\mathrm{V}$ to $\mathrm{A}$ shift. What might account for this asymmetry? One factor that may have contributed to the outcome of this experiment was the use of mixed lists. Since mixed lists have not previously been studied in this situation, some unknown aspect of that procedure may have produced the unexpected results. For this reason, another experiment was conducted. The mixed-mode filler was again employed, but independent groups were assigned to shift and control conditions learning triads of words homogeneous with respect to mode of presentation on each trial.

\section{EXPERIMENT II}

\section{Method}

Mode shift and mode of presentation on Trial 4 were orthogonally combined to yield two experimental and two control groups. One experimental group received an $\mathrm{A}$ to $\mathrm{V}$ shift (Condition AV) and the other received a $V$ to $A$ shift (Condition VA). The controls. Conditions AA and VV, did not receive a shift in mode of presentation. The apparatus, interpolated task, and general procedures were identical to those in Experiment 1 . However, the to-be-remembered materials consisted of triads rather than 4-tuples of animal names, since most of the previous research has required retention of triads. Four different lists were prepared by forming four triads of animal names and assigning each triad to a different trial in each list; the lists were counterbalanced across conditions. The $128 \mathrm{~S}$ were selected from the same pool as in Experiment $I$, and 32 were assigned to each condition according to a block-randomized procedure.

\section{Results and Discussion}

The mean numbers of words correctly recalled are presented in Fig. 2. Considering first the development of PI, the data from the first three trials were analyzed, with presentation mode. shift. and trials as factors. The only reliable source of variance was that of trials $[F(2,248)=76.35 . p<.001$. standard crror of the mean

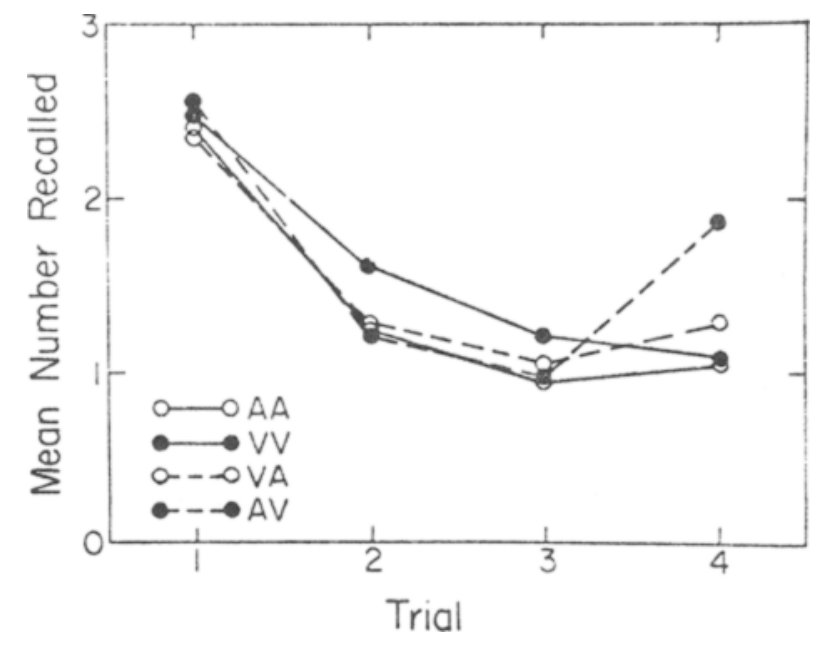

Fig. 2. Mean numbers of words correctly recalled on Trials 1-4 of Experiment II.

$=.169 \mathrm{]}$, indicating substantial development of PI. On Trial 4 there was a release from PI following an A to V shift, as evidenced by the fact that recall was better in Condition AV than in the control condition, VV $[\mathrm{t}(62)=3.93, \mathrm{p}<.001$, standard error of the mean $=$ .142]; the difference between Condition VA and its control condition, AA, was not significant $(t<1)$, indicating no reliable release from PI for a $\mathrm{V}$ to $\mathrm{A}$ shift. Thus, the asymmetrical release from PI observed in Experiment $I$ is also obtained with independent groups.

Performance on the filler task was also analyzed for this experiment and showed a slight nonmonotonic improvement in performance across trials $[\mathrm{F}(3,372)=5.96, \mathrm{p}<.001$, standard error of the mean $=.151]$. The proportions of correct responses were .87 , $.92, .87$, and .90 for Trials $1-4$, respectively. None of the other sources of variance was reliable.

\section{OVERALL DISCUSSION}

These experiments indicate that, using a mixed-mode filler, a release from PI is obtained for an $A$ to $V$ shift but not for a V to A shift. The conclusion is the same for the mixed-list procedure of Experiment I as for the independent groups design of Experiment II. The obtained asymmetry is remarkably similar to that observed when the filler task is presented in the A mode (Hopkins et al, 1973) and opposite to that obtained with a V filler (Hopkins et al, 1971, 1973). Thus, it appears that the mixed-mode filler has some of the functional characteristics of an A filler task. One factor that might have emphasized the A characteristics of the mixed-mode filler is the requirement that $\mathrm{S}$ verbalize his response to each filler item. This explanation seemed unlikely in view of the fact that Hopkins et al (1973) required a verbal response to $\mathrm{A}$ and $\mathrm{V}$ filler items but were, nevertheless. able to manipulate the direction of the asymmetry by varying the mode of presentation of the filler items. 
The potential importance of verbalization was examined by testing 24 additional Ss under conditions similar to those of Condition VM of Experiment I. The only change was to use a buttonpress instead of a verbalized response to each filler item; these Ss were to push one button if the sum of a pair of filler digits was odd and a second button if the sum was even. If verbalization of the filler responses was responsible for the failure to obtain a release from PI for a V to $A$ shift in the above experiments, then these additional Ss should have shown an abrupt improvement in recall of the A subset between Trials 3 and 4 . The mean numbers of words correctly recalled from the A subset were 1.21, $0.71,0.88$, and 0.83 for Trials $1-4$, respectively; for the $\mathrm{V}$ subset, the corresponding means were $1.25,0.83$, 0.88 , and 0.75 . Thus, these data show significant development of PI across the first three trials $[F(2,46)=3.37, p<.05$, standard error of the mean $=$ $.185]$, but recall was not significantly different for the $A$ and $V$ subsets, either over Trials 1.3 or on Trial 4 (both Fs $<1$ ). There is no indication of a release from PI for the $\mathbf{A}$ items in these data, so it appears that the mode of S's response to the filler items does not influence the release from PI. It is interesting to note that Hopkins et al (1971) reached a similar conclusion regarding verbalization of the memory items. Thus, it appears that the presentation mode is important for both memory and distractor items but that verbalization of either type of item has little effect on the release from PI following a shift in the mode of presentation of the memory items. This conclusion needs further empirical examination, since it clearly suggests that articulation by $S$ has much different effects on memory than reception of an auditory stimulus, an implication that seems to disagree with the assumptions of a number of memory theorists (e.g., Adams, 1967).

The present results are consistent with our earlier conclusion (Hopkins et al, 1973), as well as with that of Kroll, Bee, and Gurski (1973). Namely, the similarities in and differences between the representations of $A$ and $V$ items in the short-term store depend in a critical way on the mode of presentation of the rehearsal-prevention activity. On the other hand, these experiments were unsuccessful in demonstrating that a mixed-mode interpolated task would produce a symmetrical reduction in interference following a shift in mode of presentation of the memory items. Instead, the mixed-mode filler seems to have the same effect on the release-from-PI as did an $A$ filler in previous research (Hopkins et al, 1973). The reason for this fact is simply not clear. One can, of course, generate vague ad hoc hypotheses suggesting the dominance of an $A$ component in S's information processing system or that S "prefers" to process the A members of the filler items. The fact remains, however, that in order to perform at the observed $89 \%$ correct level on this filler task, S must process both $\mathrm{A}$ and $\mathrm{V}$ components.

\section{REFERENCES}

Adams, J. A. Human memory. New York: MoGraw-Hill, 1967. Battig, W. F., \& Montague, W. E. Category norms for verbal items in 56 categories: A replication and extension of the Connecticut category norms. Journal of Experimental Psychology, 1969, 80(3, Part 2).

Hopkins, R. H. A digital programmer for use with audio tape decks. Behavior Research Methods \& Instrumentation, 1972, 4, 317-319.

Hopkins, R. H., Edwards, R. E., \& Cook, C. L. Presentation modality, distractor modality, and proactive interference in short-term memory. Journal of Experimental Psychology, 1973, 98, 362-367.

Hopkins, R. H., Edwards, R. E., \& Gavelek, J. R. Presentation modality as an encoding variable in short-term memory. Journal of Experimental Psychology, 1971, 90, 319-325.

Kroll, N. E. A., Bee, J., \& Gurski, G. Proactive interference as a result of changing presentation modality. Journal of Experimental Psychology, 1973, 98, 131-137.

Wickens, D. D. Encoding categories of words: An empirical approach to meaning. Psychological Review, 1970, 77, 1-15.

Wickens, D. D. Characteristics of word encoding. In A. W. Melton and E. Martin (Eds.), Coding processes in human memory. Washington, D.C: Winston, 1972. Pp. 191-216.

(Received for publication February 5, 1973; revision received April 9, 1973.) 